\section{Gauging the importance of personalities in student, intern and resident selection}

Medical educators have become increasingly interested in the noncognitive traits and abilities of medical students and graduate physicians. Studies by Walton, Alpert, and Draba of the Chicago College of Osteopathic Medicine ${ }^{1}$ found that osteopathic medical students and practicing osteopathic physicians differed from their allopathic counterparts in possessing certain dominant personality traits.

These characteristics were measured by the Myers-Briggs Type Indicator, which is based upon the work of Carl Jung. Jung identified two ways of perceiving reality (sensing or intuition), two ways of judging reality (thinking or feeling), two ways of dealing with external reality (judging or perceiving), and two ways of being (extroverted or introverted).

The CCOM researchers studied osteopathic medical students and physicians, including osteopathic specialists, and compared the results with those from allopathic studies. They found a prominence of sensing and judging types over intuitive and perceiving personalities in osteopathic medical school freshmen and allopathic physicians interested in family medicine. Furthermore, they determined that osteopathic specialists were more like primary care allopathic physicians. In addition, osteopathic specialists shared more similar traits with their primary care colleagues than with their nonprimary care allopathic counterparts. These findings confirmed earlier work by Robert C. Ward, DO, and Howard Teitlebaum, DO, who found a similar predominance of sensing and judging types among osteopathic physicians. ${ }^{2}$

Our profession has long been characterized as one composed of "people doctors," "doctors who care." An identifiable personality type appears to be a factor in this general perception; But how heavily are personality and other nonacademic traits weighed by directors of osteopathic medical education when selecting students?

The study by Bates and colleagues, beginning on page 391 of this issue, attempts to offer some answers to this question. Among 157 directors of AOA-accredited internship and residency programs, they found that noncognitive factors (values, attitudes, and interpersonal skills) strongly. influenced the selection of interns and residents.

In general, the results indicated that nonacademic interpersonal qualities are more highly valued in osteopathic programs than in allopathic programs that were studied. In addition, interpersonal qualities were weighted more heavily in osteopathic specialty programs than they were among allopathic specialty programs.

The authors conclude that these data are consistent with the philosophy of osteopathic medicine and its emphasis on primary care. They further suggest that the emphasis on nonacademic factors in this selection process requires correlation in three areas: the program director's measurement of these criteria; students' perception of such guidelines; and the college's role in developing the qualities considered important.

As we see it, these recommendations offer some interesting grist for further discussions among our educators and possibilities for future curricula development.

THOMAS WESLEY ALLEN, DO, FACOI

\footnotetext{
1. Walton FC, Alpert JS, Draba RE: Personality characteristics of osteopathic physicians as interpreted from scores on the Myers-Briggs Type Indicator (Res Abstract). JAOA 1983;83:73.

2. Spaltro $\mathrm{K}$ : Investigators find that DOs are verifiably different. The DO $1983 ; 24: 117-120$.
} 
NEW FROM MILES

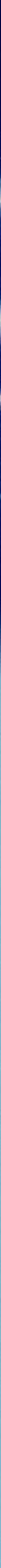




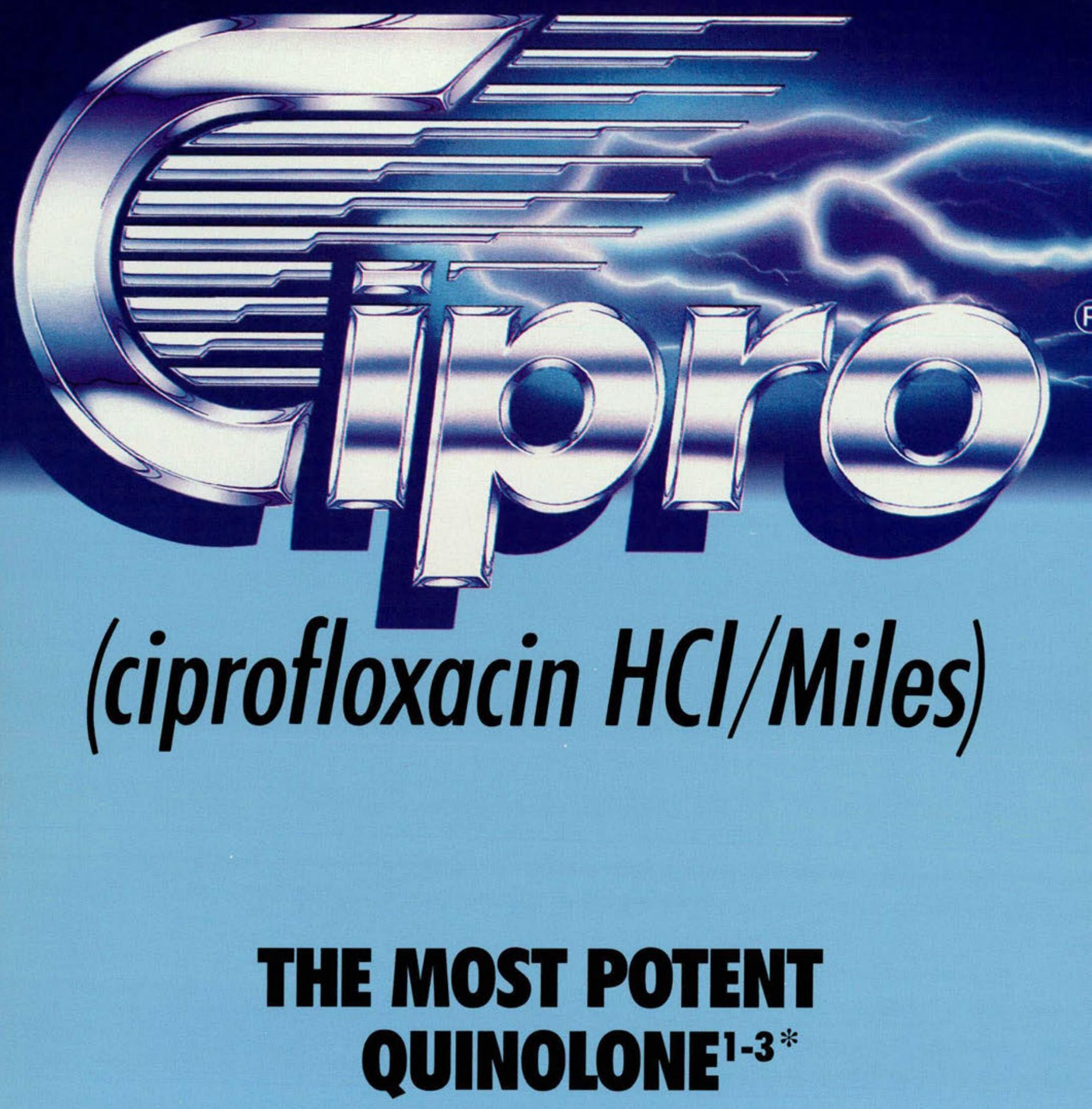

*In vitro activity does not necessarily imply a correlation with in vivo results. 


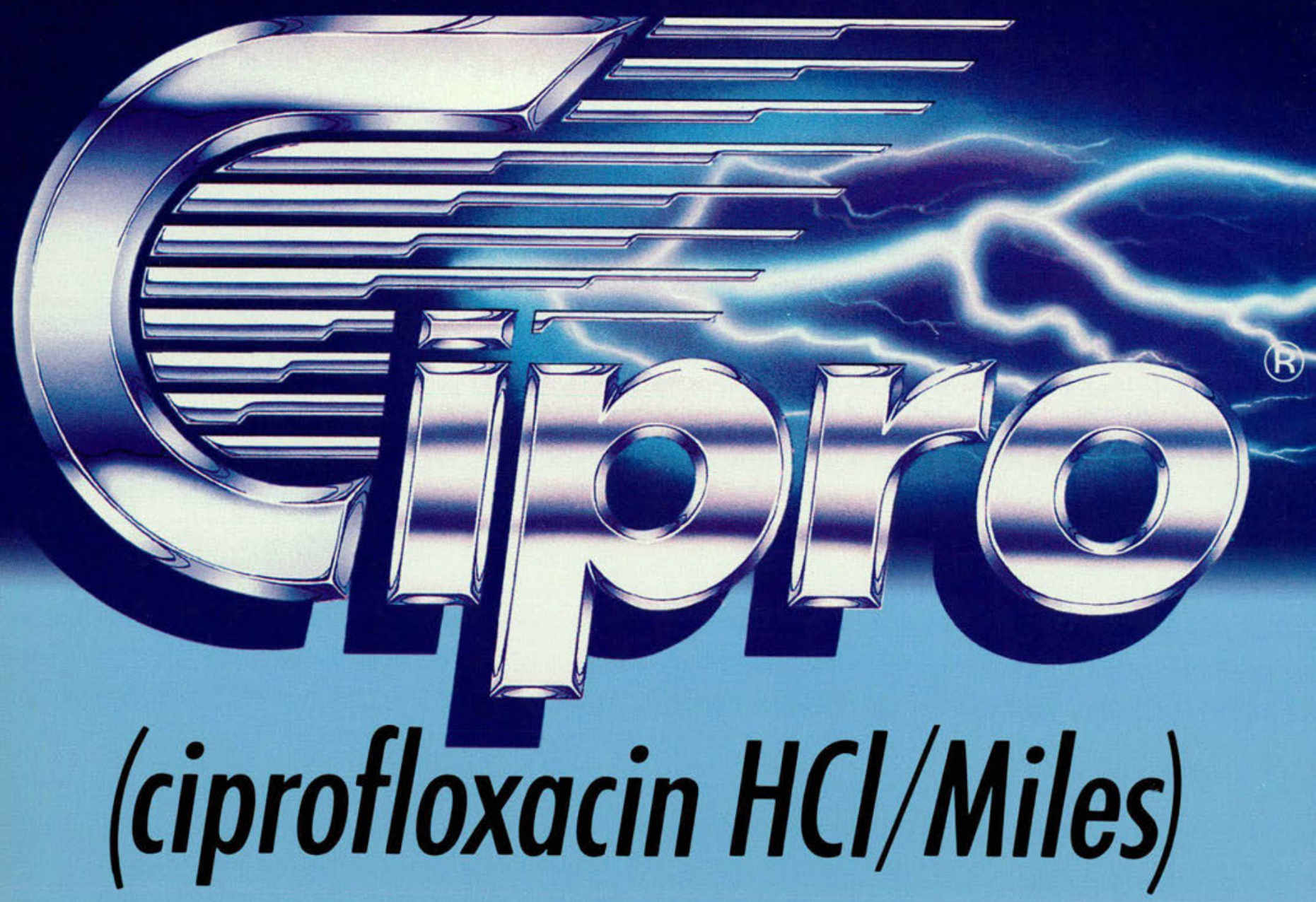



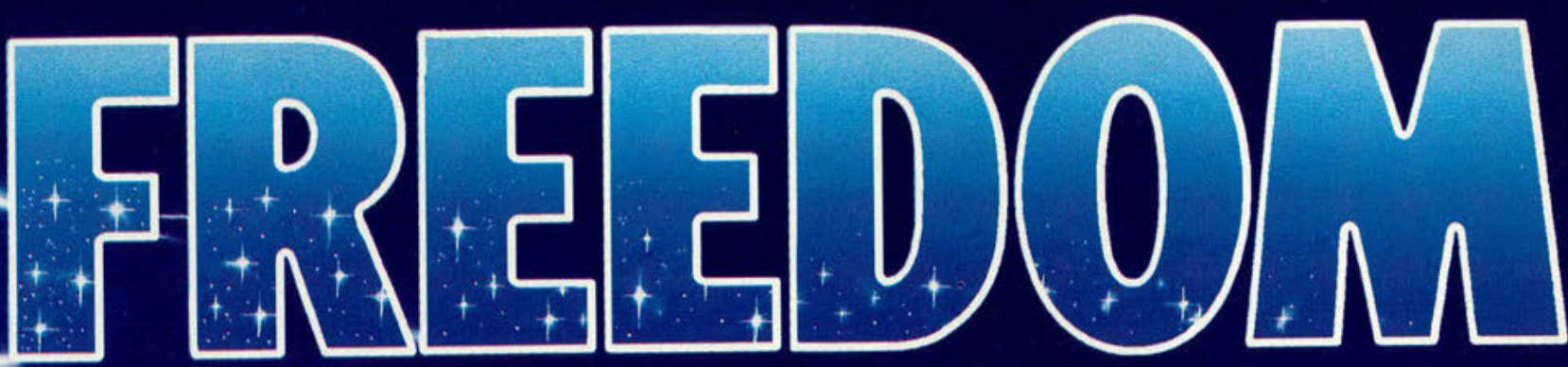

THE POWER OF PAREIIIERALS.

THE FREBDOM OF ORAL DOSAGE.

- Beyond the limitations of parenteral antimicrobials and traditional oral therapy

- For outpatient treatment of many infections that previously required hospitalization

Well tolerated-low incidence of side effects

CIPRO ${ }^{\circledR}$ SHOULD NOT BE USED IN CHILDREN OR PREGNANT WOMEN.

Notes: Concomitant administration of ciprofloxacin with theophylline may prolong the serum elimination half-life of theophylline, elevote serum

theophylline levels, and may increase the risk of adverse reactions (see precoutions).

Antocids containing mognesium hydroxide or oluminum hydroxide may interfere with the obsorption of ciprofloxacin, resulting in serum and urine levels

lower than desired: concurrent administration of these agents with ciprofloxacin should be avoided.

A history of hypersensitivity to ciprofloxacin is a contraindication to its use. A history of hypersensitivity to other quinolones may also contraindicate the use of ciprofloxocin.

For further information, contact the Miles Information Service: 1-800-642-4776.

In VA. call collect: 703-620-2799.
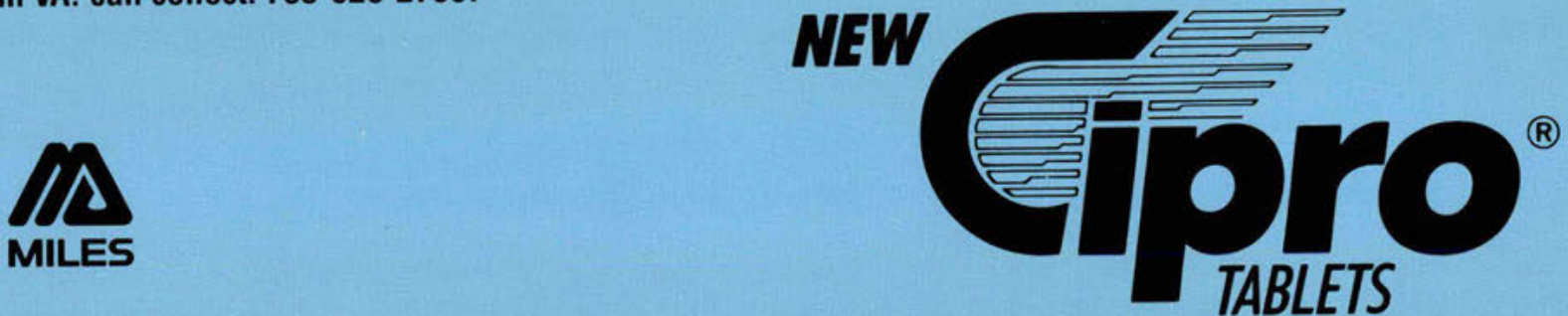
NEW

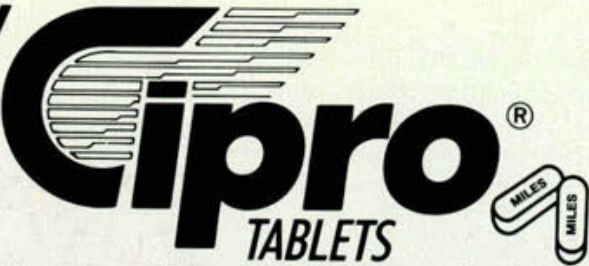 (ciprofloxacin $\mathrm{HCl} /$ Miles)}

\section{CONSULT PACKAGE INSERT FOR FULL PRESCRIBING INFORMATION INDICATIONS}

Cipro is indicated for the treatment of the following infections caused by susceptible strains of the indicated microorganisms: lower respiratory, skin and skin structure, bone and joint, and urinary tract; and infectious diarthea (SEE FULL PRESCRIBING INFORMATION FOR SPECIFIC ORGANISMS) CONTRAINDICATIONS

A history of hypersensitivity to ciprofloxacin is a contraindication to its use. A history of hypersensitivity to other

quinolones may also contraindicate the use of ciprofloxacin.
WARNINGS

CIPROFLOXACIN SHOULD NOT BE USED IN CHILDREN OR PREGNANT WOMEN. The oral administration of ciprofloxacin caused lameness in immature dogs. Histopathological examination of the weight-bearing joints of these dogs revealed permanent lesions of the cartilage. Related drugs such as nalidixic acid, cinoxacin and norfloxacin also produced erosions of cartilage of weight-bearing joints and other signs of arthropathy in immature animals of various species (SEE ANIMAL PHARMACOLOGY SECTION IN FULL. PRESCRIBING INFORMATION.

General:

\section{PRECAUTIONS}

As with other quinolones, ciprofloxacin may cause central nervous system (CNS) stimulation, which may lead to tremor, restlessness, lightheadedness, confusion, and very rarely to hallucinations or convuisive seizures. Therefore, ciprofloxacin should be used with caution in patients with known or suspected CNS disorders, such as severe cerebral arteriosclerosis or epilepsy, or other factors which predispose to seizures (SEE ADVERSE

Crystals of ciprofloxacin have been observed rarely in the urine of human subjects but more frequently in the urine of laboratory animals. Crystalluria related to ciprofloxacin has been reported only rarely in man because human urine is usually acidic. Patients receiving ciprofloxacin should be well hydrated, and alkalinity of the urine should be avoided. The recommended daily dose should not be exceeded. Alteration of the dosage regimen is necessary for patients with impairment of renal function (SEE DOSAGE AND ADMINISTRATION)

Drug Interactions:

Concurrent administration of ciprofloxacin with theophylline may lead to elevated plasma concentrations of theophylline and prolongation of its elimination half-life. This may result in increased risk of theophylline-related adverse reactions. If concomitant use cannot be avoided, plasma levels of theophylline should be monitored and dosage adjustments made as appropriate.

Antacids containing magnesium hydroxide or aluminum hydroxide may interfere with the absorption of ci. profloxacin, resulting in serum and urine levels lower than desired; concurrent administration of these agents with ciprofloxacin should be avoided

Probenecid interferes with the renal tubular secretion of ciprofloxacin and produces an increase in the level of ciprofloxacin in the serum. This should be considered if patients are receiving both drugs concomitantly

As with other broad-spectrum antibiotics, prolonged use of ciprofloxacin may result in overgrowth of nonsusceptible organisms. Repeated evaluation of the patient's condition and microbial susceptibility testing is essential. If superinfection occurs during therapy, appropriate measures should be taken.

Information for Patients:

Patients should be advised that ciprofloxacin may be taken with or without meals. The preferred time of dosing is two hours after a meal. Patients should also be advised to drink fluids liberally and not take antacids containing magnesium or aluminum concomitantly or within two hours after dosing. Ciprofloxacin may cause dizziness or lightheadedness; therefore patients should know how they react to this drug before they operate an automobile or machinery or engage in activities requiring mental alertness or coordination.

Carcinogenesis. Mutagenesis, Impairment of Fertility:

Eight in vitro mutagenicity tests have been conducted with ciprofloxacin and the test results are listed below Salmonella/Microsome Test (Negative)

E. coli DNA Repair Assay (Negative)

Mouse Lymphoma Cell Forward Mutation Assay (Positive)

Chinese Hamster $V_{79}$ Cell HGPRT Test (Negative)

Syrian Hamster Embryo Cell Transformation Assay (Negative

Saccharomyces cerevisiae Point Mutation Assay (Negative)

Saccharomyces cerevis

Rat Hepatocyte DNA Repair Assay (Positive)

Thus, two of the eight tests were positive, but the following three in vivo test systems gave negative results Rat Hepatocyte DNA Repair Assay

Micronucleus Test (Mice)

Dominant Lethal Test (Mice)

Long-term carcinogenicity studies in animals have not yet been completed.

Pregnancy-Pregnancy Category $C$

Reproduction studies have been performed in rats and mice at doses up to six times the usual daily human dose and have revealed no evidence of impaired fertility or harm to the fetus due to ciprofloxacin. In rabbits, as with most antimicrobial agents, ciprofloxacin ( 30 and $100 \mathrm{mg} / \mathrm{kg}$ orally) produced gastrointestinal disturbances resulting in maternal weight loss and an increased incidence of abortion. No teratogenicity was observed at either dose. After intravenous administration, at doses up to $20 \mathrm{mg} / \mathrm{kg}$. no maternal toxicity was produced, and no em bryotoxicity or teratogenicity was observed. There are, however, no adequate and well-controlled studies in
pregnant women. SINCE CIPROFLOXACIN. UKE OTHER DRUGS INITS CLASS, CAUSES ARTHROPATHY INIMMA. TURE ANIMALS, IT SHOULD NOT BE USED IN PREGNANT WOMEN (SEE WARNINGS).

Nursing Mothers.

It is not known whether ciprofloxacin is excreted in human milk; however, it is known that ciprofloxacin is excreted in the milk of lactating rats and that other drugs of this class are excreted in human milk. Because of this and because of the potential for serious adverse reactions from ciprofloxacin in nursing infants, a decision should be made to discontinue nursing or to discontinue the drug, taking into account the importance of the drug to the mother:

Ciprofloxacin should not be used in children because it causes arthropathy in immature animals (SEE WARNINGS)

\section{ADVERSE REACTIONS}

Ciprofloxacin is generally well-tolerated. During clinical investigation, 2,799 patients received 2,868 courses of the drug. Adverse events that were considered likely to be drug related occurred in $7.3 \%$ of courses, possibly related in $9.2 \%$, and remotely related in $3.0 \%$. Ciprofloxacin was discontinued because of an adverse event in $3.5 \%$ of courses, primarily involving the gastrointestinal system $(1.5 \%)$, skin $(0.6 \%)$, and central nervous system

The most frequently reported events, drug related or not, were nausea $(5.2 \%)$, diarrhea $(2.3 \%)$, vomiting $2.0 \%$ ), abdominal pain/discomfort $(1.7 \%)$, headache $(1.2 \%)$, restlessness $(1.1 \%)$, and rash $(1.1 \%)$

Additional events that occurred in less than $1 \%$ of ciprofloxacin courses are listed below. Those typical of quinolones are italicized.

\section{CONVENIENT B.I.D. DOSAGE}

\begin{tabular}{|c|c|c|}
\hline \multicolumn{3}{|c|}{ Recommended dosage schedule } \\
\hline Infection Site & $\begin{array}{l}\text { Severity of } \\
\text { Infection }\end{array}$ & Dosage \\
\hline \multirow{2}{*}{$\begin{array}{l}\text { Respiratory Tract } \\
\text { Bone and Joint } \\
\text { Skin/Skin Structure } \\
\end{array}$} & Mild/Moderate & $500 \mathrm{mg}$ B.I.D. \\
\hline & Severe/Complicated & $750 \mathrm{mg}$ B.I.D. \\
\hline \multirow[t]{2}{*}{ Urinary Tract } & Mild/Moderate & $250 \mathrm{mg}$ B.I.D. \\
\hline & Severe/Complicated & $500 \mathrm{mg}$ B.I.D. \\
\hline Infectious Diarrhea & Mild/Moderate/Severe & $500 \mathrm{mg}$ B.I.D. \\
\hline
\end{tabular}

GASTROINTESTINAL: (See above), painful oral mucosa, oral candidiasis, dysphagia, intestinal perfora tion, gastrointestinal bleeding

CENIALLNEAVOUSSYSTEM: (See above), dizziness, lightheadedness, insomnia, nightmares, hallucina tions, manic reaction, ifritability, tremoc, ataxia, convuisive seizures, lethargy, drowsiness, weakness, SKINUHYPERSENSITIVITY, depersonalization, depression, paresthesia

SKIN/HYPERSENSITVITY, (See above), pruritus, urticaria, photosensitivity, flushing, fever, chills, angioedema, edema of the face, neck, lips, conjunctivae or hands, cutaneous candidiasis, hyperpigmentation, erythema nodosum.

SPECIAL SENSES. blurred vision, disturbed vision (change in color perception, overbrightness of lights), decreased visual acuity, diplopia, eye pain, tinnitus, bad taste.

MUSCULOSKELETAL. joint or back pain, joint stiffness, achiness, neck or chest pain, flare up of gout: RENALUROGENITAL, interstitial nephritis, nephritis, renal failure, polyuria, urinary retention, urethra bleeding, vaginitis, acidosis

CARDIOVASCULAR: paipitations, atrial flutter, ventricular ectopy. syncope, hypertension, angina pectoris, myocardial infarction, cardiopulmonary arrest, cerebral thrombosis.

RESPIRATORY: epistaxis, laryngeal or pulmonary edema, hiccough, hemoptysis, dyspnea, bronchospasm, pulmonary embolism

Most of these events were described as only mild or moderate in severity, abated soon after the drug was discontinued, and required no treatment

In several instances, nausea, vomiting, tremor, restlessness, agitation, or paipitations were judged by investigators to be related to elevated plasma levels of theophylline possibly as a result of a drug interaction with ci-

Adverse Laboratory Changes: Changes in laboratory parameters listed as adverse events without regard to drug relationship:

Hepatic-Elevations of: ALT (SGPT) (1.9\%), AST (SGOT) (1.7\%). Alkaline Phosphatase $(0.8 \%), 10 \mathrm{HH}(0.4 \%)$ serum bilirubin $10.3 \%$ :

Hematologic-eosinophilia $(0.6 \%)$, leukopenia $(0.4 \%)$, decreased blood platelets $(0.1 \%)$, elevated blood platelets $(0.1 \%)$, pancytopenia $(0.1 \%)$.

Cenal-Eevations of. Serum creatinine $(1.1 \%)$, BUN $(0.9 \%)$

CRYSTALLURIA, CYLINDRURIA, AND HEMATURIA HAVE BEEN REPORTED.

Other changes occurring in less than $0.1 \%$ of courses were: Elevation of serum gammaglutamyl transferase, elevation of serum amylase, reduction in blood glucose, elevated uric acid, decrease in hemoglobin, anemia, bleeding diathesis, increase in blood monocytes, and leukocytosis.
OVERDOSAGE

Information on overdosage in humans is not available. In the event of acute overdosage, the stomach should be emptied by inducing vomiting or by gastric lavage. The patient should be carefully observed and given supportive treatment. Adequate hydration must be maintained. In the event of serious toxic reactions from overdosa tive modialysis or peritoneal dialysis may aid in the removal of ciprofloxacin from the body. particularly if renal function is compromised

\section{DOSAGE AND ADMINISTRATION}

The usual adult dosage for patients with urinary tract infections is $250 \mathrm{mg}$ every 12 hours. For patients with complicated infections caused by organisms not highly susceptible, $500 \mathrm{mg}$ may be administered every 12 hours. Respiratory tract infections, skin and skin structure infections, and bone and joint infections may be treated with $500 \mathrm{mg}$ every 12 hours. For more severe or complicated infections, a dosage of $750 \mathrm{mg}$ may be given every 12 hours

The recommended dosage for infectious diarrhea is 500 mg every 12 hours.

In patients with renal impairment, some modification of dosage is recommended ISEE DOSAGE AND ADMIN HOW SUPPUED

Cipro* (ciprofloxacin HCl/Miles) is available as tablets of $250 \mathrm{mg} .500 \mathrm{mg}$, and $750 \mathrm{mg}$ in bottles of 50 , and in Unit-Dose packages of 100 (SEE FULL PRESCRIBING INFORMATION FOR COMPLETE DESCRIPTION).

References: 1. Barry, A.L., Jones, R.N., Thornsberry, C., et al.; Antibacterial activities of ciprofloxacin, norfloxacin, oxolinic acid, cinoxacin, and nalidixic acid. Antimicrob. Agents Chemother. 25:633-637, 1984, 2. Guimaraes, M.A., and Noone, P. The comparative in-vitro activity of norfloxacin, ciprofloxacin, enoxacin and nalidixic acid against 423 strains of gram-negative rods and staphylococci isolated from infected hospitalised patients. J. Antimicrob. Chemother. 17:63-67, 1986. 3. Van Caekenberghe, D.L., and Pattyn, S.R.: In vitro activity of ciprofloxacin compared with those of other new fluorinated piperazinyl-substituted quinoline derivatives. Antimicrob. Agents
Chemother. 25:518-521, 1984.

For further information, contact the Miles Information Service: 1-800-642-4776. In VA. call collect: 703-620-2799.

\section{COMMITTED TO THERAPEUTIC EFFICIENCY}

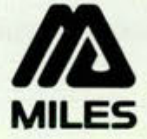

c) November 1987, Miles Inc.
Miles Inc

Pharmaceutical Division 400 Morgan Lane West Haven, CT 06516 